\title{
LOCALLY AFFINE SPACES WITH NILPOTENT FUNDAMENTAL GROUPS
}

\author{
BX \\ J. C. SANWAL
}

1. Introduction. In this paper we consider compact locally affine spaces with nilpotent fundamental groups and show that such spaces are homeomorphic to compact nilmanifolds.

In order to state our results more precisely, we introduce the following definitions and notation which will be used throughout this paper. Let $E^{n}$ be the $n$-dimensional vector space over the real field and let $\Lambda$ be a set of automorphisms of $E^{n}$. Then, as usual, we shall write for $C \otimes E^{n}$ and $1 \otimes \Lambda$, $E_{(C)}^{n}$ and $\Lambda_{(C)}$ respectively, where $C$ is the field of complex numbers. If the set of automorphisms $\Lambda$ of $E^{n}$ has the form

$$
\left(\begin{array}{llll}
\Lambda_{1} & & & 0 \\
& \Lambda_{2} & & \\
& & \ddots & \\
0 & & & \Lambda_{p}
\end{array}\right)
$$

in some matrix representation, we shall denote it by $\Lambda_{1}+\Lambda_{2}+\cdots+\Lambda_{p}$. Let $X$ be an $n$-dimensional locally affine space, i.e., a compact differentiable manifold with a given complete affine connection with curvature and torsion zero. It is known, Auslander and Markus [1], that $\pi_{1}(X)=\Gamma$ can be imbedded as a discrete subgroup of the group of affine transformations $A(n)$ of the $n$ dimensional affine plane and furthermore, $E^{n}$ is a universal covering space over $X \approx E^{n} / \Gamma$ relative to the natural identification map $p ; p(v)=\Gamma v$ for $v \in E^{n}$.

Using homogeneous coordinates we can consider $\Gamma$, with the above imbedding, as a group of automorphisms of $E^{n+1}$. We shall prove that $\Gamma_{(c)}$ is indecomposable and, an easy consequence of this fact is that, if $\Gamma$ is a nilpotent group it is unipotent, i.e., each element of $\Gamma$ has all its eigenvalues equal to 1 . Therefore, by Theorem 1 of Wang [8], there exists a connected, simply connected nilpotent Lie group $N$ containing $\Gamma$ as a uniform, discrete subgroup. We shall finally show that $X$ is homeomorphic to $N / \Gamma$.

Our central result is:

A locally affine space $X$ with nilpotent fundamental group $\Gamma$ is homeomorphic to the nilmanifold with fundamental group $\Gamma$, i.e., the homogeneous space of a connected, simply connected nilpotent Lie group by the uniform, discrete subgroup $\Gamma[5]$.

Presented to the Society, August 30, 1960; received by the editors September 13, 1960 and, in revised form, March 15, 1961. 
I take this opportunity to thank Dr. L. Auslander for his encouragement and suggestions.

2. Spaces with a discrete operator group. We shall consider quite general structures in this section, even though in later applications we shall need only particular cases of the results obtained here.

Let $G$ be a discrete group acting on a topological manifold $M$ (connected and separable) without fixed points such that $M / G$ is a topological manifold. Let

$$
R=\{(x, y): x, y \in M ; g x=y \text { where } g \in G\}
$$

be the graph of $G$. Then $(M, M / G, p)$ is a principal fibre space (see Séminaire H. Cartan 1949/50) if $\phi: R \rightarrow G$, defined by $\phi(x, g x)=g$, is continuous.

The principal fibre space $(M, M / G, p)$ is locally trivial if and only if there is a local cross-section at each point of $M / G$. Now, the continuity of $\phi$ is equivalent to

(1) There is an open neighbourhood $U_{1}$ of $m_{1}$ for each $m_{1} \in M$ such that $U_{1} \cap g U_{1}=\varnothing$ for $g \neq 1$ (1 will always denote the identity element of the group).

Then it is easy to see that the map $s_{1}: p\left(U_{1}\right) \rightarrow U_{1}$, defined in the obvious manner, provides a local cross-section. Hence $(M, M / G, p)$ has local product structure and $M$ is a covering space of $M / G$. Conversely, a universal covering space is a principal fibre space in the above sense.

Defnition 2.1. Let $(M, M / G, p)$ be a principal fibre space. A subspace $D$ of $M$ is called a fundamental domain relative to $G$ if

(i) Map $p \mid D$ is bijective;

(ii) $D$ is a countable union of locally closed subsets of $M$.

The following lemma is quite well known and was proved for the case when $M$ is a topological group and $G$ a discrete subgroup of $M$ by Siegel [7]. We give the proof for completeness.

LEMma 2.1. For the principal fibre space $(M, M / G, p)$ there always exists a fundamental domain $D$. Further, if $M / G$ is compact then $D^{-}$is compact and conversely.

Proof. Let

$$
v=\left\{V_{i}: p\left(U_{i}\right)=V_{i} \text { and } U_{i} \text { satisfy the condition (1) }\right\}
$$

be a countable covering of $M / G$.

Define

$$
\begin{aligned}
& D_{1}=s_{1}\left(V_{1}\right), \\
& D_{n}=s_{n}\left(V_{n}\right)-\left[\bigcup_{1}^{n-1} p^{-1}\left(V_{i}\right)\right], \quad n=2,3, \cdots,
\end{aligned}
$$


where $s_{i}$ are the sections defined by $U_{i}$ and

$$
D=\bigcup_{1}^{\infty} D_{n}
$$

If $x \in M / G$ then there is a smallest integer $n$ such that

$$
x \in V_{n}-\left[\begin{array}{c}
n-1 \\
\bigcup_{1}
\end{array} V_{i}\right]
$$

and hence $s_{n}(x) \in D_{n}$. Therefore $p \mid D$ is surjective. Let $d_{1}, d_{2} \in D$ such that $p\left(d_{1}\right)=p\left(d_{2}\right)$. Then both $d_{1}$ and $d_{2}$ cannot be in $D_{i}$ due to condition (1). Suppose $j<i$ and $d_{1} \in D_{j}$ and $d_{2} \in D_{i}$ then

$$
D_{i} \cap g D_{j} \subset\left[\bigcup_{1}^{i-1} p^{-1}\left(V_{l}\right)\right]-\left[\bigcup_{1}^{i-1} p^{-1}\left(V_{l}\right)\right]=\varnothing
$$

Hence $p \mid D$ is an injection.

Let us suppose now that $M / G$ is compact. We can assume that the covering $V$ is finite. Since $M$ is locally compact, $\left[s_{i}\left(V_{i}\right)\right]$ - can be assumed to be compact. Therefore

$$
D^{-}=\overline{D_{1}} \cup \overline{D_{2}^{-}} \cup \cdots \cup \overline{D_{r}^{-}}
$$

is compact, as each $D_{i}^{-}$is compact. Conversely, if $D^{-}$is compact then $p\left(D^{-}\right)$ $=M / G$ is compact.

Using the above lemma we can now prove

Lemma 2.2. Let $(M, M / G, p)$ be a principal fibre space, where $M$ has dimension $n$ with $\pi_{i}(M)=0, i \geqq 1$ and $M / G$ is compact. Then there does not exist a simply connected, aspherical closed submanifold $C$ of $M$ of dimension strictly less than $n$, which is invariant under $G$.

Proof [2]. Since $M / G$ is compact by Lemma 2.1 there exists a fundamental domain $D$ for $M$ relative to $G$ with compact closure. Suppose to the contrary that such a $C$ exists. Therefore $C \cap D$ is a fundamental domain for $C$ relative to $G$ such that $[C \cap D]-$ is compact. Hence $C / G$ is a compact manifold by Lemma 2.1, with fundamental group $G$. We shall compute the $n$th cohomology group of $G$ in two ways and get the required contradiction. We have

$$
H^{n}(G, Z /(2)) \cong H^{n}(C / G, Z /(2)),
$$

and

$$
H^{n}(G, Z /(2)) \cong H^{n}(M / G, Z /(2)),
$$

by Theorem 1 of [4]. Now $H^{n}(C / G, Z /(2))=0$ for $\operatorname{dim} C / G<n$ and $H^{n}(M / G, Z /(2))=Z /(2)$ because with $Z /(2)$ as coefficient group the Pontrja- 
gin duality theorem remains valid for nonorientable manifolds. Hence the lemma follows.

3. Nilpotent matrix groups. It is known that if $A$ and $B$ are two automorphisms of a vector space $V$ over the complex field and

$$
[B[\cdots[B[B A]] \cdots]]=0 \text {, where }[B A]=B A-A B,
$$

then $A$ leaves the characteristic subspaces of $B$ invariant, i.e., $\{v: v \in V$ and $(B-\lambda I)^{m} v=0$ for some $\left.m\right\}$, where $\lambda$ is an eigenvalue of $B$. The following lemma is a partial group analogue of the above result.

LEMMA 3.1. Let $\sigma, \tau$ be two automorphisms of a complex vector space such that

$$
(\tau(\cdots(\tau(\tau \sigma)) \cdots))=I \text {, where }(\tau \sigma)=\tau^{-1} \sigma^{-1} \tau \sigma .
$$

If $\sigma$ and $\tau$ are triangulable, i.e., they can be put in triangular form simultaneously, then $\sigma$ leaves the characteristic subspaces of $\tau$ invariant. Furthermore, in a certain basis,

$\sigma=\sigma_{1} \dot{+} \cdots \dot{+} \sigma_{r}, \sigma_{i}$ are triangular matrices,

$\tau=\tau_{1} \dot{+} \cdots \dot{+} \tau_{r}, \tau_{i}$ are triangular matrices with $\tau_{i}=c_{i} \times$ unipotent matrix and if $i \neq j, c_{i} \neq c_{j}$.

Proof. We shall prove the lemma by induction on the order of the matrices. It is trivially true for $n=1$. Suppose the lemma has been proved for matrices of order $\leqq n-1$.

In some basis of the vector space, $\sigma$ and $\tau$ have the form

$$
\sigma=\left(\begin{array}{cc}
a & s \\
0 & \sigma^{*}
\end{array}\right), \quad \tau=\left(\begin{array}{ll}
b & t \\
0 & \tau^{*}
\end{array}\right),
$$

where $\sigma^{*}, \tau^{*}$ are triangular matrices and $a, b$ are the eigenvalues of $\sigma, \tau$ respectively. Since $\sigma^{*}, \tau^{*}$ satisfy all the conditions of the lemma, we have by hypothesis

$$
\sigma=\left(\begin{array}{ccccc}
a & s_{1} & s_{2} & \cdots & s_{r} \\
& \sigma_{11} & & 0 \\
& & \sigma_{22} & & \\
0 & & & & \sigma_{r r}
\end{array}\right), \quad \tau=\left(\begin{array}{lllll}
b & t_{1} & t_{2} & \cdots & t_{r} \\
& \tau_{11} & & 0 \\
& & \tau_{22} & \\
0 & & & & \tau_{r r}
\end{array}\right)
$$

where $\sigma_{i i}, \tau_{i i}$ are triangular matrices with $\tau_{i i}=b_{i} \times$ unipotent matrix. Now, there are two possibilities; either $b_{i}=b$ for some $i$ or $b_{i} \neq b$ for all $i$. In both cases the proof proceeds along the same lines. Therefore, we shall give the proof in detail for the first case, when $b_{i}=b$ for some $i$. Without loss of generality, we can assume that $i=1$. Then, there exists a matrix 


$$
A=\left(\begin{array}{ccccc}
1 & 0 & x_{2} & \cdots & x_{r} \\
& I & & & 0 \\
& & A_{2} & & \\
& & & \ddots & \\
& & & & A_{r}
\end{array}\right)
$$

with $A_{i}$ triangular, such that

$$
\tau^{\prime}=A \tau A^{-1}=\left(\begin{array}{lllll}
b & t_{1} & 0 & \cdots & 0 \\
& \tau_{11} & & & 0 \\
& & \tau_{2} & & \\
& & & \ddots & \\
& & & & \tau_{r}
\end{array}\right) .
$$

Notice that $\tau_{i}$ are triangular matrices.

To complete the proof it is sufficient to show that

$$
A \sigma A^{-1}=\left(\begin{array}{lllll}
a & s_{1} & 0 & \cdots & 0 \\
& \sigma_{11} & & & 0 \\
& & \sigma_{2} & & \\
& & & \ddots & \\
& & & & \sigma_{r}
\end{array}\right)
$$

with $\sigma_{i}$ triangular. Let us suppose to the contrary that

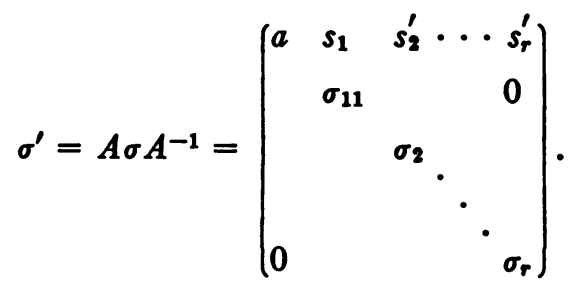

Now

$$
\begin{aligned}
& \eta\left(\tau^{\prime}, \sigma^{\prime}\right)=\left(\cdots\left(\tau^{\prime}\left(\tau^{\prime} \sigma^{\prime}\right)\right) \cdots\right) \\
& =\left(\begin{array}{ccccc}
1 & \phi_{1} & \phi_{2}^{\prime} & \cdots & \phi_{r}^{\prime} \\
& \eta_{11} & & 0 \\
& & \eta_{2} & & \\
& & & \ddots & \\
0 & & & & \eta_{r}
\end{array}\right),
\end{aligned}
$$


where $\phi_{i}^{\prime}$ are polynomials in $a, b, s_{i}^{\prime}, \sigma_{i}, \tau_{i}$ and $\phi_{1}$ in $a, b, s_{1}, \sigma_{11}, \tau_{11}$ and $\eta_{i}=\eta\left(\tau_{i}, \sigma_{i}\right)$. Then

$$
\left(\tau^{\prime}, \eta\left(\tau^{\prime}, \sigma^{\prime}\right)\right)=\left(\begin{array}{cccc}
1, & * & , \cdots, b^{-1} \phi_{r}^{\prime} \eta_{r}^{-1}\left[b I-\tau_{r}\right] \eta_{r} \\
& & \ddots & 0 \\
0 & & \ddots & \left(\tau_{11}, \eta_{11}\right) \\
& & \\
0 & & \left(\tau_{r}, \eta_{r}\right)
\end{array}\right) .
$$

Therefore, if $\phi_{i}^{\prime} \neq 0$ then $b^{-1} \phi_{i}^{\prime} \eta_{i}^{-1}\left[b I-\tau_{i}\right] \eta_{i} \neq 0$, but this contradicts (2), hence $s_{i}^{\prime}=0$.

Remark 1. From the proof of Lemma 3.1, it is obvious that if $\Sigma$ is a set of automorphisms such that each element of $\Sigma$ and $\tau$ satisfy (2) and if $\Sigma$ and $\tau$ are triangulable then, in some basis,

$\Sigma=\Sigma_{1} \dot{+} \cdots+\Sigma_{r}$, each $\alpha \in \Sigma_{i}$ is triangular, for all $i$,

$\tau=\tau_{1} \dot{+} \cdots \dot{+} \tau_{r}, \tau_{i}$ are triangular matrices with $\tau_{i}=c_{i} \times$ unipotent matrix and if $i \neq j, c_{i} \neq c_{j}$.

COROLlary 3.2. If $N$ is a nilpotent complex matrix group then there exists a normal subgroup $N^{\prime}$ of finite index in $N$ which is reducible to the form $N^{\prime}=N_{1}+\cdots+N_{p}$. Further, if $\alpha_{1}+\cdots+\alpha_{p} \in N^{\prime}$ then $\alpha_{i}$ is scalar plus nilpotent, i.e., $\alpha_{i}=b I+S$ where $b$ is a complex number and $S$ is a strictly triangular matrix.

Proof. Mal'cev [6] has shown that a solvable complex matrix group has a triangulable subgroup of finite index. Hence there is a triangulable normal subgroup $N^{\prime}$ of finite index in $N$, for intersection of a finite number of subgroups of finite index has itself finite index. Existence of a triangulable normal subgroup $N^{\prime}$ can also be proved by using the Lie-Kolchin theorem, then of course one does not need Mal'cev's result. Let us suppose then that the result is true for matrices of order $\leqq n-1$. If each matrix of $N^{\prime}$ have all its eigenvalues equal, then we are done. Otherwise, let $\tau \in N^{\prime}$ have $r$ different eigenvalues then by Lemma $3.1, N^{\prime}=N_{1}^{\prime} \dot{\cdots} \cdots+N_{r}^{\prime}$, where $N_{i}^{\prime}$ is triangular. Since order of each member of $N_{i}^{\prime}$ is $<n$, we have the desired result by induction hypothesis.

The following lemma is a generalization of Lemma 6 of $\mathrm{Mal}^{\prime} \mathrm{cev}$ [5]. As a word of caution we add that the hypothesis in the following lemma is such that if $\Lambda$ is a group then the lemma can be applied only when it is a unipotent group.

Lemma 3.3. Let $\Lambda=\Lambda_{1}^{\prime}+\cdots+\Lambda_{r}^{\prime}$ be a set of automorphisms of a complex vector space, where $\Lambda_{i}^{\prime}, i=1, \cdots, r$; are in the triangular form. Further assume that every element of $\Lambda_{i}^{\prime}$ has all its eigenvalues equal to $a_{i}$ and any two elements of $\Lambda_{i}^{\prime}$ have the same eigenvalue $a_{i}$ and if $i \neq j, a_{i} \neq a_{j}$. Let $\tau$ be an automorphism of the complex vector space such that $\tau^{-1} \Lambda \tau=\Lambda$ and for all $\alpha \in \Lambda$, let 
$(\tau \cdots(\tau(\tau \alpha)) \cdots)=I$. Then $\tau$ and $\Lambda$ can be simultaneously reduced to the following form:

$\tau=\tau_{1} \dot{+} \cdots+\tau_{k}$, where $\tau_{i}$ is scalar plus nilpotent,

$\Lambda=\Lambda_{1}+\cdots+\Lambda_{k}, \Lambda_{i}$ are in the triangular form and every element of $\Lambda_{i}$ has all its eigenvalues equal to $b_{i}$.

Proof. Let $V$ be the complex vector space on which $\Lambda$ acts. Then $V=V_{1}^{\prime}+\cdots+V_{r}^{\prime}$ and each $V_{i}^{\prime}$ is left invariant by $\Lambda$. Notice

$$
V_{i}^{\prime}=\left\{v: v \in V \text { and }\left(\alpha-a_{i} I\right)^{n_{i}} v=0 \text { for all } \alpha \in \Lambda\right\},
$$

where $n_{i}=\operatorname{dim} V_{i}^{\prime}$. Because $\tau^{-1} \Lambda \tau=\Lambda$ it follows that $\tau V_{i}^{\prime} \subset V_{i}^{\prime}$ for $\left(\alpha-a_{i} I\right)^{n_{i}} \tau v$ $=\tau\left(\tau^{-1} \alpha \tau-a_{i} I\right)^{n_{i}}=0$. Hence $\tau=\tau_{1}^{\prime} \dot{+} \cdots+\tau_{r}^{\prime}$. Therefore it is sufficient to show that the lemma is true for $\tau_{i}^{\prime}$ and $\Lambda_{i}^{\prime}$.

Let $\gamma=\tau_{i}^{\prime}, \theta=\Lambda_{i}^{\prime}$. Then $\gamma$ and $\theta$ satisfy the conditions of the lemma. Define $W^{i}=\left\{w: w \in V_{i}^{\prime}\right.$ and $\left(\alpha-a_{i} I\right)^{i} w=0$ for all $\left.\alpha \in \theta\right\}$, then $W^{1} \subset W^{2} \subset \cdots$ $C W^{n_{i}}=V_{i}^{\prime}$. Since $\gamma^{-1} \theta \gamma=\theta, \gamma$ leaves each $W^{j}$ invariant. Choose a basis of $V_{i}^{\prime}$ consisting of bases of $W^{i}$ such that $\gamma$ and $\theta$ are simultaneously triangular in this basis. Lemma 3.1 now proves the result.

The following proposition is valid for group $\Gamma$, not necessarily nilpotent, and shall be used to prove Theorem 3.5 later on.

Proposition 3.4. If $\Gamma$ is the fundamental group of a locally affine space then $\Gamma_{(C)}$ is indecomposable.

Proof. Let $\left(1 \otimes e_{1}, \cdots, 1 \otimes e_{n} ; 1 \otimes e_{n+1}\right)$ be a basis of $E_{(C)}^{n+1}$ in which elements of $\Gamma_{(C)}$ have the matrix representation

$$
\left(\begin{array}{ll}
g & t \\
0 & 1
\end{array}\right), \quad g \in G L(n) .
$$

Let us suppose to the contrary that, $E_{(C)}^{n+1}=W_{1}+W_{2}$, where $W_{i}$ are invariant subspaces with bases $\left(f_{1}, \cdots, f_{k}\right),\left(f_{k+1}, \cdots, f_{n+1}\right)$. We may assume without loss of generality that $f_{1}=\sum g_{1 j} \cdot 1 \otimes e_{j}$ such that $g_{1 n+1} \neq 0$. Then the linear subspace generated by $f_{1}$ intersects the hyperplane $\left[x_{1}+i y_{1}, \cdots, x_{n}+i y_{n}, 1+i\right]$ where $i^{2}=-1$, with respect to the basis $\left(1 \otimes e_{1}, \cdots, 1 \otimes e_{n} ; 1 \otimes e_{n+1}\right)$, of $E_{(C)}^{n+1}$ in a point $b$. If we identify the above hyperplane with $E_{(C)}^{n}$ by the natural map

$$
\left[x_{1}+i y_{1}, \cdots, x_{n}+i y_{n}, 1+i\right] \rightarrow\left(x_{1}+i y_{1}, \cdots, x_{n}+i y_{n}\right),
$$

we can write $b=b_{1}+i b_{2}$ where $b_{i} \in E^{n}$. With the above identification in mind, suppose that $\Gamma b_{i}$, for $i=1,2$; spans an $r$-dimensional subspace of $E^{n}$, then by Lemma 2.2, $r$ must be equal to $n$. But this implies that $\Gamma_{(C)} b$ spans $E_{(C)}^{n}$. Therefore $\Gamma_{(C)} f_{1}$ spans $E_{(C)}^{n+1}$, i.e., $W_{2}=0$. This completes the proof of the proposition.

THEOREM 3.5. Let $X$ be a locally affine space with nilpotent fundamental group $\Gamma$. Then $\Gamma$ as a matrix group is unipotent. 
Proof. To prove the theorem it is sufficient to show that $\Gamma_{(C)}$ is unipotent. By Corollary 3.2, there is a normal subgroup $\Delta$ of finite index in $\Gamma$ which is reducible to the form

$$
\Delta_{1}+\cdots \dot{+} \Delta_{p}
$$

Now $\left(E^{n} / \Delta, E^{n} / \Gamma, \pi\right)$ is a principal fibre space with structural group $\Gamma / \Delta$. As $E^{n} / \Gamma$ is compact and $\Gamma / \Delta$ finite it follows that $E^{n} / \Delta$ is compact and again $E^{n} / \Delta$ is a locally affine space. Therefore from Proposition 3.4 it follows that $\Delta$ is indecomposable and hence $p$ must be equal to 1 . Since $\Delta \subset A(n)$, each element of $\Delta$ has at least one eigenvalue equal to 1 and hence $\Delta$ is unipotent.

Finally, suppose there is an element $\tau \in \Gamma$ which is not unipotent. As $\tau$ and $\Delta$ satisfy all the conditions of Lemma 3.3 we conclude that $\Delta$ is decomposable, which is a contradiction. Hence $\Gamma$ must be unipotent.

4. Proof of the main theorem. Theorem 3.5 implies that the nilpotent fundamental group $\Gamma$ of $X$ can be put simultaneously in the triangular form with 1's along the main diagonal (Kolchin). Hence there exists a connected, simply connected nilpotent linear Lie group $N \subset A(n)$, containing $\Gamma$ as a uniform subgroup (see Theorem 1, Wang [8]). Further, $\Gamma$ is a discrete subgroup of $N$ because $\Gamma$ is a discrete subgroup of $A(n)$. Since $\Gamma$ is a fundamental group of an aspherical, compact $n$-dimensional manifold, it easily follows from Theorem 1 of [4], that $\operatorname{dim} N=n$.

Let us define a map $\eta: N \rightarrow E^{n}$ by

$$
\eta\left(\begin{array}{ll}
g & t \\
0 & 1
\end{array}\right)=t
$$

This map $\eta$ was investigated by Auslander [3] and it follows as a particular case of his Lemma 7 that $\eta$ is a homeomorphism. However, we shall give an outline of the proof in our case following his method of proof.

It follows by a straight forward calculation that $\eta\left(n_{1} n_{2}\right)=n_{1} \eta\left(n_{2}\right)$ where $n_{1}, n_{2} \in N$. Now $\eta(N)=N / K$ (left coset space) where $K$ is a closed connected subgroup of $N$ which consists of elements of the form $g+1$. Considering part of the homotopy sequence of the fibering $p: N \rightarrow N / K$

$$
\rightarrow \pi_{1}(N) \rightarrow \pi_{1}(N / K) \rightarrow \pi_{0}(K) \rightarrow
$$

we conclude that $\pi_{1}(N / K)=0$. From the theorem of Chevalley which states that, $N / K$ is homeomorphic to the cartesian product of circles and straight lines, it now follows that $N / K$ is homeomorphic to a Euclidean space. Hence $\eta(N)$ is a closed subset of $E^{n}$. Since $\Gamma$ leaves $\eta(N)$ invariant, we conclude from Lemma 2.2 that $\eta(N)=E^{n}$. Since $\operatorname{dim} N=\operatorname{dim} \eta(N), K$ must be the identity matrix. Therefore $N \approx E^{n}$ and from $\eta\left(n_{1} n_{2}\right)=n_{1} \eta\left(n_{2}\right)$ it follows that $N / \Gamma \approx E^{n} / \Gamma$. Hence we have 
THEOREM 4.1. If $X$ is a locally affine space with nilpotent fundamental group then $X$ is homeomorphic to a nilmanifold.

An obvious corollary of this theorem is that if two locally affine spaces have isomorphic nilpotent fundamental groups then they are homeomorphic.

\section{REFERENCES}

1. L. Auslander and L. Markus, Holonomy of flat affinely connected manifolds, Ann. of Math. (2) 62 (1955), 139-151.

2. L. Auslander, On the group of affinities of locally affine spaces, Proc. Amer. Math. Soc. 9 (1958), 471-473.

3. - Some compact solvmanifolds and locally affine spaces, J. Math. Mech. 7 (1958), 963-976.

4. S. Eilenberg and S. MacLane, Relation between homology and homotopy groups of spaces, Ann. of Math. (2) 46 (1945), 480-509.

5. A. I. Mal'cev, On a class of homogeneous spaces, Amer. Math. Soc. Transl. 39 (1951), $12-589$.

6. - On some classes of infinite soluble groups, Amer. Math. Soc. Transl. (2) 2 (1956), 1-21.

7. C. L. Siegel, Discontinuous groups, Ann. of Math. (2) 44 (1943), 674-689.

8. H. C. Wang, Discrete subgroups of solvable Lie groups. I, Ann. of Math. (2) 64 (1956), 1-19.

CORNELl UnIVERSity,

ITHACA, NEW YORK 